\title{
e-Migrinter
}

22 | 2021

Varia

\section{Les subalternes de l'asile : Pourquoi certains récits sont inaudibles}

Karine Gatelier

\section{CpenEdition}

Journals

Édition électronique

URL : https://journals.openedition.org/e-migrinter/2654

DOI : 10.4000/e-migrinter.2654

ISSN : 1961-9685

Éditeur

UMR 7301 - Migrinter

Référence électronique

Karine Gatelier, «Les subalternes de l'asile : Pourquoi certains récits sont inaudibles », e-Migrinter [En ligne], 22 | 2021, mis en ligne le 25 novembre 2021, consulté le 17 décembre 2021. URL : http:// journals.openedition.org/e-migrinter/2654; DOI : https://doi.org/10.4000/e-migrinter.2654

Ce document a été généré automatiquement le 17 décembre 2021.

Tous droits réservés 


\title{
Les subalternes de l'asile : Pourquoi certains récits sont inaudibles
}

\author{
Karine Gatelier
}

\section{Introduction}

1 Le 13 juillet 2017, M.C., originaire de la Gambie, commence le récit des causes de son exil et de sa demande d'asile. Nous sommes dans un petit bureau de la Maison des Associations de Grenoble, je suis bénévole d'une association ${ }^{1}$ et accompagne les personnes en demande d'asile depuis 2010. Ce jour-là, je prépare M.C. à l'entretien auquel il est convoqué à l'office français de Protection des Réfugiés et Apatrides (OFPRA) où sa demande d'asile est examinée. M.C. s'exprime dans sa langue maternelle ; il est venu avec un ami qui parle bien français et fait la traduction pour lui. J'ai quitté mon pays parce que j'ai des problèmes de famille. Je suis fils unique et ma mère est morte.

2 M.C. avait alors 36 ans. Son père est décédé plusieurs années auparavant. Ce dernier avait une seconde femme, et de ce second mariage sont nés plusieurs enfants, ses demifrères et sœurs. Dès le décès de sa mère, ces derniers l'ont chassé de la maison familiale pour en prendre possession, en dépit des règles de l'héritage, et M.C. affirme qu'ils cherchent maintenant à le tuer. M.C. a bien essayé de porter plainte auprès des policiers mais ceux-ci favorisent ses demi-frères, et ne le protègent ni ne défendent ses droits. Face à la résistance de M.C., les demi-frères l'ont menacé de mort à plusieurs reprises.

3 Le récit de M.C. s'arrête à peu près ici. Il raconte des détails, en réponse à mes questions, sur les conditions dans lesquelles ses demi-frères l'ont menacé de mort, décrivant les moyens utilisés, les formes que ces menaces ont prises. Mais je comprends qu'en l'état, ce récit est insuffisant pour les critères de l'OFPRA : ces éléments ne rendent pas assez réelles les menaces. Je multiplie les questions sur les pratiques de l'héritage, sur la fréquence dans le pays de ce genre d'assassinats pour s'emparer d'un héritage, sur les soutiens que M.C. aurait trouvés etc. mais mes questions ne trouvent 
pas vraiment de réponse et semblent surprendre M.C. Bref, notre échange dure et je ne parviens pas à trouver d'éléments dont je sais qu'ils sont attendus par l'officier de l'OFPRA. Je pressens déjà comment cette demande d'asile sera reçue à l'OFPRA: " évasive ", "sans réalité ", " aux propos non circonstanciés et impersonnels ", selon une terminologie bien connue. En revanche, j'en apprends davantage sur l'existence et la position sociale de M.C. dans son pays. Je comprends qu'il n'avait ni travail ni salaire régulier et parvenait à gagner un maigre revenu en ramassant les ordures dans le village avec un tracteur qui ne lui appartenait pas. Je comprends aussi que pour porter plainte auprès de la police, il a dû se rendre à la ville voisine à pied parce qu'il n'a pas les moyens de payer un transport ni d'amis à qui demander ce service. Tous ces détails m'informent que M.C. ne peut compter ni sur un revenu ni sur le soutien d'un réseau de relations. Enfin, j'observe sa difficulté à comprendre et s'exprimer, au vu des échanges laborieux avec son ami qui traduit pour lui. Finalement, et parce que je me rends soudain compte que M.C. ne m'a pas donné cette information, je lui demande comment est morte sa mère. M.C. répond simplement : Mes demi-frères l'ont tuée.

Ce n'est qu'après plus d'une heure d'échanges que M.C. donne une information essentielle pour rendre crédible la menace qu'il a fuie, et seulement en réponse à ma question. Si ses demi-frères ont tué sa mère, alors leurs menaces à l'égard de M.C. peuvent être plus convaincantes. Pourquoi M.C. n'a-t-il pas saisi que cette information est essentielle? Son ami qui l'accompagne s'adresse à lui avec autorité, et semble lui donner des conseils. M.C. fait des réponses très succinctes à mes questions. Je vois qu'il lui est difficile de raconter ce qui s'est passé, ce qu'est sa vie au village. Pourtant il s'exprime dans sa langue maternelle et son ami maîtrise bien le français.

5 Avec cette histoire, fondatrice dans ma démarche de recherche, et emblématique de certaines demandes d'asile, je voudrais poser la question de la capacité de certaines personnes en demande d'asile à parler, dans le contexte de l'examen de leur demande, au regard des attentes en matière de récit, des modalités du recueil de celui-ci, de la nature de l'échange et l'écoute: permettent-ils à toutes les personnes en demande d'asile de s'exprimer et d'être entendues? Il s'agit donc de comprendre ce qui empêche cette prise de parole.

\section{Méthode et cadre théorique}

6 En citant l'histoire de M.C., je propose de questionner pourquoi il n'a pas donné une information capitale pour comprendre la réalité de la situation face à laquelle il se trouvait et des menaces qui le visaient ? Au-delà du cas de M.C., je voudrais développer l'hypothèse que toutes les personnes en demande d'asile ne peuvent pas parler et être entendues, dans l'examen de leur demande d'asile. Pour cela, je m'appuierai sur d'autres situations rencontrées dans l'accompagnement bénévole à différentes étapes de la procédure d'asile et plus particulièrement sur les préparations à l'entretien de l'OFPRA. Dans ce cadre, j'ai assisté à plusieurs entretiens à l'OFPRA en tant que «tiers accompagnant ", je lis les notes d'entretiens et les décisions motivées de l'OFPRA, et dans les années précédentes, je rédigeais les recours auprès de la Cour nationale du Droit d'Asile (CNDA). Cet accompagnement se poursuit depuis 2010, parfois de manière irrégulière, en fonction de ma disponibilité. Je ne choisis pas le profil des personnes que je reçois, puisque c'est le fait des hasards du planning de l'association. A ces entretiens, s'ajoute le suivi de quelques personnes que j'accompagne tout au long de leur 
procédure, depuis mes autres bénévolats dans d'autres collectifs. Ainsi, j'observe les procédures de personnes avec lesquelles je reste en lien ou au contraire d'autres que je ne vois qu'une seule fois ; c'est pourquoi, sur la durée, il m'a permis de découvrir une variété de situations. Enfin, cette réflexion s'appuie sur des entretiens menés avec d'autres bénévoles de l'association et avec des professionnels. En revanche aucun entretien de recherche n'a été réalisé avec des personnes en demande d'asile, à cause de leur configuration trop asymétrique et du fait qu'il reproduirait trop les entretiens de la procédure.

7 Le cas de M.C. a été particulièrement éloquent car, si son histoire permettait de reconnaître certains des critères bien connus des attendus du jugement de l'asile, le fait qu'il ne les fournisse pas spontanément m’a intriguée. En amont de cet événement, je portais un intérêt pour les Subaltern studies et plus particulièrement pour l'approche de Spivak, présentée dans son ouvrage Les subalternes peuvent-elles parler? (2009). Mon entretien avec M.C. m'a directement renvoyée à l'analyse de Spivak.

Spivak s'inscrit dans la continuité des travaux de Gramsci ${ }^{2}$ et de Guha ${ }^{3}$ qui font le constat de l'absence des groupes subalternes dans l'historiographie qualifiée d'élitiste par ce dernier et posent la question d'une méthode pour écrire cette historiographie manquante. Mais Spivak critique la démarche à deux égards : comment écrire l'histoire des subalternes alors que les seules archives disponibles sont celles de colons? Une telle entreprise comporte de plus un risque d'essentialisation de la condition subalterne. Spivak (2009) définit les sujets subalternes comme les victimes paradigmatiques de la division internationale du travail. Ce ne sont pas seulement les opprimés; ce sont celles et ceux qui sont tellement déplacées qu'ils et elles manquent d'organisation et de représentation politique, les deux éléments-clé de la subalternité. Une fois organisés et représentés politiquement, les subalternes cessent d'être subalternes. Elle voit dans cette position sociale un obstacle à faire entendre leur parole : les subalternes ne peuvent être entendu'es sans utiliser le code et le langage de leurs oppresseurs car l'énonciation de la norme et des formes de subjectivités étant fixées par les dominants, toute autre narration reste inaudible. Quand les subalternes sont représentées, ils et elles le sont comme l'Autre dans l'idéologie dominante, dans une altérité à la fois radicale et homogénéisante.

9 Ainsi, la condition de certaines personnes en demande d'asile peut correspondre à la condition de subalternes quand elles partagent ce déficit d'organisation et de représentation politique pour se faire entendre. L'existence d'un savoir hégémonique sur l'asile qui agit comme un cadre dans lequel les demandes sont jugées évoque également cette condition. Enfin, l'individualisation de la demande d'asile a renforcé cette situation en créant une grande inégalité entre les personnes, en fonction de leur capital social qui détermine leur capacité à se faire entendre. La difficulté à prendre la parole est donc analysée comme un indicateur de la réalité de cette position subalterne. Elle peut être observée dans l'impossibilité de se saisir de la parole, de contredire ou de dire non aux personnes, bénévoles ou professionnelles, qui les aident. L'OFPRA agit comme un instrument de l'hégémonie, il est donc encore plus difficile d'y prendre la parole. Les personnes en demande d'asile se contentent de répondre aux questions, et quittent parfois le bureau sans avoir raconté leur histoire.

L'hypothèse de cette recherche repose sur l'idée que, dépossédées de leur parole, les personnes en demande d'asile sont des sujets subalternes. Et elle consiste à comprendre tout ce qui parle à leur place. Elle élargit donc le cadre au-delà des institutions de 
l'asile, et des relations avec les personnes qui les incarnent. Il s'agit de chercher à comprendre les constructions mentales et les représentations qui expliquent que certaines paroles ne sont pas entendues.

11 Cette question a été le point de départ d'une autre démarche d'action-recherche qui a pris la forme d'un atelier-radio pour ouvrir un espace de parole protégé pour les personnes en demande d'asile ${ }^{4}$.

12 La demande d'asile est une injonction à se raconter, à construire un récit de soi singulier puisqu'il doit correspondre aux attentes de celles et ceux qui jugent l'asile. De nombreux travaux ont étudié certains obstacles rencontrés par les demandeurs d'asile pour être entendus (1). Cet article propose d'ajouter l'analyse de tout ce qui parle à la place des personnes en demande d'asile et précède la formulation de leur récit (2). Dans cette analyse, la violence épistémique est un concept important et on verra comment elle s'impose aux personnes en demande d'asile, les dépossédant de leur parole et faisant d'elles des sujets subalternes (3).

\section{Le récit empêché}

L'injonction à se raconter, que représente la demande d'asile, enserre l'émergence du récit de soi dans une diversité d'obstacles dont de nombreux travaux rendent compte.

Le régime d'asile institué par la convention de Genève de 1951 évolue dans le sens d'une individualisation de la demande, quand jusque-là il se fondait sur l'appartenance à une communauté persécutée. Même si la nationalité continue de jouer un rôle important, déterminé par la nature des relations diplomatiques qui désignent des nationalités plus ou moins désirables en fonction des périodes (Akoka, 2020), cette individualisation a imposé que chaque personne en demande d'asile produise son récit propre. L'instruction repose dès lors sur les capacités de chaque individu à argumenter, à prouver, à convaincre. Ainsi s'instaure un faisceau d'inégalités entre les personnes (D'Halluin, 2007) dans cette soumission à l'épreuve de la crédibilité (Kobelinsky, 2007 ; Fassin, 2013).

Les questions linguistiques, celles de la traduction et des différences culturelles sont un registre supplémentaire qui est source de malentendus, de mauvaises interprétations et d'incompréhensions (D'Halluin, 2007). De plus et généralement, les personnes en demande d'asile ont une connaissance faible du cadre juridique dans lequel s'inscrit leur parole; c'est pourquoi ils et elles sont accompagnées dans cette mise en récit par des travailleurs sociaux, des juristes ou des bénévoles d'associations qui font un travail de médiation mais aussi d'interprétation. Ainsi le récit est tout autant la production des aidantes que celle des personnes en demande d'asile, et revient à un «travail de conformation " (D'Halluin, 2004), créant ainsi une dépendance des personnes en demande d'asile vis-à-vis des personnes qui les aident. L'enjeu est donc celui de retranscrire l'histoire pour se conformer à ces attentes. Cette mise aux normes est une source supplémentaire d'interprétation du vécu et de dépossession.

La méconnaissance des attentes de celles et ceux qui jugent l'asile peut parfois conduire à faire de mauvais choix comme par exemple en dissimulant ou en réprimant des émotions (Freedman, 2017). En effet, le système d'examen de l'asile impose des contraintes fortes lors des confrontations de la parole des personnes en demande d'asile avec celles et ceux qui la jugent (Freedman, 2017 ; D'Halluin, 2004) : les questions 
souvent courtes se succèdent à un rythme soutenu et déstructurent le récit ${ }^{5}$, bien souvent déjà perçu comme suspect (Valluy, 2004; Fassin et Kobelinsky, 2012). La rédaction du récit connaît bien souvent une limite en nombre de pages, du fait du coût de la traduction. Le temps de l'entretien est de plus en plus court, sous l'injonction au raccourcissement de la procédure ${ }^{6}$. Ces deux dernières contraintes ont des effets directs sur la difficulté, et parfois l'impossibilité, de reconstruire la menace originelle qui a poussé à l'exil et qui met des années à se constituer ; c'est un effet d'accumulation de détails et d'événements dans la vie qui finissent par pousser au départ. Or il n'y a pas de place pour une telle "expansion narrative" (Valluy, 2004). Enfin, les dernières réformes de l'asile concourent toutes à raccourcir la durée de la procédure, forçant ainsi les personnes en demande d'asile à formuler leur récit de plus en plus tôt après leur arrivée ${ }^{7}$. Ces éléments souvent étudiés constituent certains des obstacles qui empêchent certaines personnes en demande d'asile de formuler leur récit.

Enfin plusieurs auteures notent que les années 1980 marquent un tournant dans la conception de l'asile avec une inversion du taux de protection: de 80 à $90 \%$ jusque dans les années 70 , le taux se situe entre 15 et $30 \%$ dans les décennies qui suivent (Akoka, 2020). Il conduit à construire l'équation que les déboutés sont des "faux réfugiés » (Fassin et Kobelinsky, 2012), ce qui permet de légitimer le refus d'accueil (Grégoire, 2008). A cela s'ajoute, au début des années 80, une transformation lexicale avec l'apparition du terme «demandeur d'asile ». D'une part elle oriente l'examen de l'asile vers une logique de sélection en mettant les personnes à distance de la figure de réfugié ; d'autre part, elle pose le problème de leur nombre et subordonne l'asile aux politiques de réduction des flux migratoires (Akoka, 2020).

Loin de reposer sur des définitions précises permettant des distinctions claires, l'asile est le produit d'interprétations politiques et de convictions personnelles, créant en réalité un rapport de force (Valluy, 2004 ; Akoka, 2020). C'est là l'origine de l'émergence dans le débat public de la figure du "faux réfugié $»^{8}$ et la transformation de l'économie morale de l'asile qui repose désormais sur la fiction d'une définition claire et d'un cadre précis. L'asymétrie que crée le système est de plus renforcée par le déclassement social violent et la précarité matérielle qui frappent les demandeurs d'asile, leur imposant la condition indigente de devoir recourir à la charité (Freedman, 2017); et de n'avoir bien souvent pas d'hébergement. L'asymétrie du rapport de force est alors abyssale.

\section{Ce qui parle à la place des personnes en demande d'asile}

Le contexte contemporain de l'asile montre que, parmi les personnes qui le sollicitent, certaines sont entravées dans ce droit par manque de ressources sociales pour formuler leur récit et par une dépendance à des aidant-es qui les influencent. L'étroitesse de l'espace qui leur est offert pour exprimer les fondements de leur demande de protection, par la condition misérable qui leur est réservée et par l'hostilité que leur renvoie le soupçon ambiant à leur égard réduisent la capacité des personnes en demande d'asile à prendre des initiatives et choisir des stratégies de manière autonome. A ces analyses existantes, il faut ajouter celle d'un ensemble de représentations et de connaissances qui agit avant l'émergence du récit des personnes en demande d'asile et, les précédant, parle à leur place. Il s'agit de la connaissance produite en Europe sur les contextes de guerre, de conflits et de violences d'origine des 
demandeurs d'asile, composée de la médiatisation qui en est faite ainsi que des études et analyses spécialisées. Il concerne également et plus largement les représentations qu'on a de l'asile.

La médiatisation des conflits agit comme une sorte d'hyper-texte qui parle à la place des personnes en demande d'asile, et avant elles. La nature de cette médiatisation détermine les représentations disponibles sur les contextes fuis et fait que la réception du récit d'asile dépendra de sa coïncidence ou pas avec l'idée préalablement construite. Un exemple frappant est celui de l'Érythrée. Longtemps restée méconnue, l'Érythrée a suscité l'intérêt croissant des journalistes et des chercheur.es au point que dès 2015, il était possible d'entendre que les Érythréens sont éligibles au statut dès que les autorités sont certaines "qu'il s'agit de «vrais "Érythréens" ". Dans ce cas, la médiatisation aide à être entendu'es. Mais en sens inverse, la médiatisation d'un conflit peut en donner une lecture exclusive et ne plus laisser de place pour d'autres types de persécutions. Par exemple, une femme originaire du Darfour a vu sa vie menacée par ses supérieurs hiérarchiques, dans le cadre de son emploi, car elle a dénoncé leur corruption. Ceux-ci se trouvaient être des gradés de l'armée. Cette situation, qui a mis en péril sa famille toute entière, concerne bien peu le conflit du Darfour qui oppose des milices du régime de Omar el-Béchir à des communautés d'éleveurs. Pourtant, son entretien à l'OFPRA a été consacré à déterminer à quelle ethnie elle appartient pour la ranger d'un côté ou de l'autre de ce conflit, vu en France à travers une grille de lecture ethnique. Pour l'officier, la priorité était de déterminer ses relations avec la rébellion darfouri. Ne laissant d'espace pour le conflit et les persécutions qu'elle a subies de ses chefs militaires, sa demande a été rejetée par l'OFPRA.

21 Dans le cas présent, non seulement la médiatisation mais aussi la construction de la connaissance sur les conflits orientent les questions d'entretien vers les situations connues et il devient alors difficile d'évoquer d'autres situations si on ne possède pas la confiance en soi et la maîtrise du langage. On peut y voir une homogénéisation du sujet victime de persécutions, et l'impossibilité, pour certaines personnes, d'être entendues pour d'autres causes de persécutions. Ainsi il apparaît que la position sociale de la personne en demande d'asile lui donnera ou pas l'aisance d'insérer des informations annexes aux questions posées. De plus, l'intimidation face à la situation est grande et permet rarement de s'écarter des sujets soulevés par les questions de l'officier. Il n'est pas rare que l'officier n'accepte tout simplement pas un changement de sujet initié par le demandeur et le condamne : «C'est moi qui pose les questions ici », s'est entendu dire un demandeur à l'OFPRA10.

22 Au statut social est lié une certaine vision élitiste de l'asile qui demeure encore aujourd'hui. Ainsi, les journalistes et les autres professions intellectuelles, les cadres de partis politiques et les autres formes d'activisme sont des situations traditionnellement assimilées à l'asile qui situent ces demandeurs dans un cadre connu et familier. A l'opposé, les pratiques normatives voire violentes, les régulations de conflits présentés comme traditionnelles, notamment dans les cas de succession et d'héritage, n'ont pas été historiquement identifiées comme des causes de besoin de protection. Dans ces situations, les personnes en demande d'asile ne peuvent compter que sur elles-mêmes pour transmettre à l'officier la réalité qu'elles ont vécue. Alors que le journaliste n'a pas besoin d'expliquer les enjeux de la pratique de son métier.

«Dans l'imaginaire français, le réfugié est celui qui a mis sa vie en péril pour des principes contre un pouvoir politique despotique (...) principes qui s'apparentent aux valeurs de la République » (Grégoire, 2008, p.72). 
, on peut observer combien le corps parle à la place du sujet. En effet, les stigmates des mauvais traitements peuvent constituer un élément important pour rendre plus crédible un récit. Ainsi le corps et le savoir expert des médecins, à travers les diagnostics et attestations qu'ils et elles font, sont des éléments largement mobilisés dans la demande d'asile. Les rapports de plaidoyer des organisations non gouvernementales ou internationales font eux aussi appel au savoir médical pour donner plus de crédit à leur analyse des situations. Faire parler les corps est une opération qui homogénéise et standardise la représentation des personnes en demande d'asile et en cela occulte la parole singulière. Écouter des experts pour faire parler les corps plutôt que le sujet efface encore davantage les personnes en demande d'asile en tant que sujets. Ainsi on assiste à une substitution de la personne qui porte la parole : le médecin et non plus la personne qui a vécu les faits. En mettant à distance le récit singulier, les personnes en demande d'asile disparaissent derrière la foule des victimes et s'effacent derrière la parole des experts.

Si un corpus de connaissances constitué dans les sociétés européennes et si des experts tirant des conclusions sur l'état des corps des personnes en demande d'asile parlent à la place de ces dernières, il est possible d'y voir une violence épistémique.

\section{La violence épistémique dans le jugement de l'asile}

La violence épistémique est un concept issu d'une lecture décoloniale des rapports de force créés et perpétués par les enjeux de la production du savoir. Elle repose sur l'existence imposée d'un centre émetteur de normes épistémiques, héritée de la domination coloniale. Le savoir occidental est valorisé et érigé en référence, reléguant les formes non-occidentales du savoir. Edward Said nous rappelle que les deux fondamentaux de l'autorité coloniale sont le pouvoir et la connaissance (1980), créant des hiérarchies tenaces que les indépendances n'ont finalement pas remises en cause ; la décolonisation des esprits restant à faire (Mignolo, 2001).

Les études occidentales font de l'Occidental le sujet de l'Histoire et du non-Occidental son objet. Dépossédés du pouvoir de dire qui ils et elles sont, la subalternité a à voir avec la lutte pour se faire entendre (Spivak, 2009) ; se faire reconnaître comme capables d'expliquer et d'analyser leurs propres expériences et non seulement comme seuls témoins comme les désignent les scientifiques occidentaux :

« No need to hear your voice when I can talk about you better than you can speak about yourself. No need to hear your voice. Only tell me about your pain. I want to know your story. And then I will tell it back to you in a new way. Tell it back to you in such a way that it has become mine, my own. Re-writing you I write myself anew. I am still author, authority. I am still colonizer, the speaking subject and you are now at the center of my talk $»^{11}$ (hooks, 1990, p.343).

Comme il a été démontré plus haut, l'examen de la demande d'asile est une épreuve de la crédibilité (Fassin et Kobelinsky, 2012) où la parole des personnes cherchant une protection est remise en cause. Un demandeur d'asile après son entretien à l'OFPRA en témoigne : Vous nous demandez de raconter notre histoire et après vous nous croyez pas ${ }^{12}$. Les décisions de rejet de l'OFPRA en effet sont pleines de formules qui mettent en doute la véracité des faits et la réalité des situations évoquées, donnant l'impression que les agents les connaissent mieux. Par exemple, une demande d'asile invoquant des persécutions subies en Albanie du fait des orientations sexuelles du demandeur est 
rejetée au prétexte qu'un rapport de mission de l'OFPRA dans ce pays a pu établir qu'il existe une organisation de défense des droits LGBTI... or par crainte de représailles, elle doit se cacher et donc rester très largement inconnue des personnes concernées. Ainsi on comprend que les connaissances produites par l'institution ne peuvent être discutées ou mises en perspectives par les informations apportées par les personnes en demande d'asile. Ces derni-ères ne peuvent donc pas être vu'es comme émetteur.trice d'un savoir à partager.

Tout ceci démontre que les personnes qui jugent l'asile produisent de la connaissance sur les situations de persécution et énoncent le réel, parlant à la place des personnes en demande d'asile; que les jugements sont fondés sur des connaissances géopolitiques produites en Occident et par conséquent incomplètes et biaisées, notamment une médiatisation sélective.

Les témoignages d'officiers de l'OFPRA montrent qu'ils et elles sont formées à poser des questions objectives comme la géographie du pays, la chronologie des événements politiques, le nom des personnalités etc. qui sont des types de savoir auquel ils et elles sont formées dès les premières années de scolarisation. Spivak nous explique comment "l'épistémè accomplit sa fonction de programmation silencieuse » (Spivak, 2009, p.43) en imposant une façon de rendre compte de la réalité et condamnant toute autre forme de savoir au rang de mensonge. Une ancienne officier de l'OFPRA fait part de son désarroi au cours de sa formation quand elle assiste à un entretien où l'officier décide de couper court sans écouter le récit de la personne parce qu'elle n'a su répondre à aucune question sur la géographie de son pays. Si elle ment sur sa provenance géographique, pourquoi étudierait-il son dossier $?^{13}$ souligne-t-elle.

30 L'ensemble de la connaissance produite par les sociétés d'accueil sur les contextes d'origine des personnes en demande d'asile agit comme une violence épistémique parce qu'elle est hégémonique et sert à discréditer les récits des personnes, parce qu'elle s'impose, les empêchant de se dire et d'être entendues.

31 Le temps de l'entretien à l'OFPRA est cadré par une trame de questions préparée par l'officier, sur la base du récit envoyé. Pour des raisons d'efficacité et par manque de temps, ils et elles suivent cette trame et sont réticentes à des interventions des personnes en demande d'asile qui couperaient le fil de leur questionnaire. Par conséquent, l'entretien suit le cours prévu par l'officier, sur la base du récit envoyé. Plusieurs témoignages de personnes demandant l'asile et d'accompagnants montrent que lorsque ceux ou celles-ci veulent prendre la parole, on les fait taire. Une phrase fréquemment entendue dans de tels cas est:Monsieur, vous êtes là pour collaborer. Pourtant, l'enjeu du temps à la disposition des personnes est central pour disposer de leur capacité à se raconter. Contraint-es par le cadrage de l'entretien par l'officier, ils et elles sont empêchées de se le réapproprier pour exprimer librement leurs craintes.

Comme le montrent les notes d'entretien envoyées avec la décision de l'OFPRA, les entretiens se terminent systématiquement par une question rituelle, devenue réglementaire, sur la volonté de la personne d'ajouter des informations qu'elle n'aurait pas pu donner. Pourtant on observe que cette question apporte rarement des éléments importants pour la demande d'asile. Elle vient à la fin de l'entretien, la personne en demande d'asile a été accablée pour toutes les questions précédentes, et éventuellement par l'attitude de l'officier ; elle semble arriver trop tard. Elle n'a pas de qualité véritable. En revanche, elle constitue un risque car sans permettre aux personnes de prendre la parole, elle verrouille le recours devant la Cour Nationale du 
Droit d'Asile (CNDA) car l'OFPRA peut dire que la question ayant été posée, la personne aurait pu se saisir de ce moment. Il est donc plus difficile d'utiliser comme argument qu'elle n'a pas eu l'occasion de s'exprimer. Cet argument postule que les circonstances devraient permettre la libre expression de la personne interrogée par l'officier de l'OFPRA. Or les intimidations sont nombreuses : poids de l'institution et de son pouvoir de décider de l'avenir de la personne ; l'officier perçue comme un'e expertee ; son statut de représentant·e de l'État.

\section{Conclusion et implications pour nos pratiques de chercheur'es}

Sans mes questions patientes à M.C. il ne m'aurait pas dit que sa mère a été tuée par ses demi-frères. M.C. ne fait que répondre à mes questions. Dans mon rôle de bénévole, accompagnant la demande d'asile dans une association, je me perçois dans une complicité avec lui. Pourtant son silence n'indique-t-il pas qu'il me voit plutôt dans une position plus proche de celle qui vont le juger? Que je suis identifiée au système hégémonique? Alors que je me ressens dans une position de transfuge ${ }^{14}$.

La relation d'aide et d'accompagnement, tout comme la configuration de recherche, posent et perpétuent des relations de domination, avec les personnes aidées, accompagnées et enquêtées. Sans questionner cette relation par une attitude réflexive sur nos positions et sur les privilèges et les soubassements de la société qui l'ont construite, le travail d'accompagnement et de recherche ne fera que reproduire l'ordre établi.

Désormais je comprends que ce qui n'est pas dit à l'OFPRA n'est pas seulement tu parce que dur à dire, émotionnel ou douloureux. Tout ce qui n'y est pas dit est l'effet de la structure hégémonique du savoir : toutes les personnes en demande d'asile n'ont pas la parole.

\section{BIBLIOGRAPHIE}

Agier, Michel (2002) Au bord du monde, les réfugiés, Paris, Flammarion

Akoka, Karen (2020) L'asile et l'exil. Une histoire de la distinction réfugiés/migrants, Paris, La Découverte.

Akoka, Karen ; Clochard Olivier et Tcholakova, Albena (2017) « Éditorial : La condition de réfugiée : expériences subjectives et mobilisations collectives ", Revue européenne des migrations internationales, vol. $33, \mathrm{n}^{\circ} 4$, pp. 7-21.

D’Halluin, Estelle (2004) « Comment produire un discours légitime? », Plein droit, n 63, pp. 30-33

D'Halluin, Estelle (2007) « Travailler sa voix ou comment rendre sa demande d'asile audible », REVUE Asylon(s), $\mathrm{n}^{\circ} 2$, Terrains d'ASILES, en ligne : http://www.reseau-terra.eu/article660.html 
De Certeau, Michel (1994) La prise de parole et autres écrits politiques, édition établie et présentée par Luce Giard, Paris, Le Seuil.

Fassin, Didier (2013) « The Precarious Truth of Asylum », Public Culture, vol. 25, n¹, pp. 39-63

Fassin, Didier et Kobelinsky, Carolina, (2012) « Comment on juge l'asile. L'institution comme agent moral », Revue française de sociologie, vol. 53, n4, pp. 657-688.

Belkis, Dominique ; Franguiadakis, Spyros ; Jaillardon, Edith et Sylvie, Bernigaud (2002) «L'aide aux demandeurs d'asile - La part du mouvement associatif dans l'accès à l'asile », rapport du CRESAL

Freedman, Jane (2017) « Peur, honte, humiliation? Les émotions complexes des demandeurs d'asile et des réfugiés en Europe », Migrations Société, n¹03, pp. 31-39

Gatelier, Karine (2017) « La prise de parole peut-elle émanciper de la position victimaire ? » in Claske Dijkema et Morgane Cohen (dir.), Cahier des $2^{e}$ Rencontres de géopolitique critique, Le droit à la ville, Mars 2017 http://www.irenees.net/bdf_fiche-experience-831_fr.html

Grégoire, Vincent (2008) «Migrants et réfugiés, ou la reconnaissance comme tri », Sens-dessous, vol. $2, \mathrm{n}^{\circ} 4$, pp. $67-79$

Green, Marcus (2002) « Gramsci cannot speak : Presentaions and interpretations of the Gransci's concept of the subaltern », Rethinking Marxism, vol.14, $\mathrm{n}^{\circ} 3$, pp. 1-24.

Greslier, Florence (2007) « La Commission des Recours des Réfugiés ou 'l'intime conviction' face au recul du droit d'asile en France », Revue Européenne des Migrations Internationales, vol. 23, n², pp. 107-133.

Grosfoguel, Ramón ; Cohen, Jim (2012) « Un dialogue décolonial sur les savoirs critiques entre Frantz Fanon et Boaventura de Sousa Santos », Mouvements, $n^{\circ} 72$, pp. 42-53

hooks, bell, (1990) Yearning : race, gender, and cultural politics, Boston, South End Press.

Kobelinsky, Carolina (2007) Le jugement quotidien des demandeurs d'asile. Recueil Alexandries, Collection Esquisses, http://reseau-terra.eu/article559.html

Makaremi, Chowra (2008) « Participer en observant. Étudier et assister les étrangers aux frontières » in Fassin, Didier ; Bensa, Alban, Les politiques de l'enquête. Epreuves ethnographiques, pp. 165-183

Mekdjian, Sarah (2016) «Les récits migratoires sont-ils encore possibles dans le domaine des Refugees Studies ? Analyse critique et expérimentation de cartographies créatives ", ACME: An International Journal for Critical Geographies, vol.15, $\mathrm{n}^{\circ} 1$, pp. 150-86.

Mignolo, Walter (2001) « Géopolitique de la connaissance, colonialité du pouvoir et différence coloniale », Multitudes, vol.3 n6, pp. 56-71.

Noiriel, Gérard (2005) État, nation et immigration. Vers une histoire du pouvoir, Paris, Gallimard. Quijano, Anibal (2007) « 'Race’ et colonialité du pouvoir », Mouvements, n51, pp. 111-118 Rancière, Jacques (2003) « La scène révolutionnaire et l'ouvrier émancipé : 1830-1848 », Tumultes, $\mathrm{n}^{\circ} 20$

Said, Edward, W (1980) L'orientalisme. L'Orient créé par l'Occident, Paris, Le Seuil.

Spivak, Gayatri Chakravorty (2009) Les subalternes peuvent-elles parler? Paris, Éditions Amsterdam. Valluy, Jérôme (2004) « La fiction juridique de l'asile », Plein droit, n63, pp. 17-22 
Valluy, Jérôme (2008) « Du retournement de l'asile (1948-2008) à la xénophobie de gouvernement : construction d'un objet d'étude », Cultures \& Conflits [En ligne], ${ }^{\circ} 69$, mis en ligne le 17 juin 2008, consulté le 03 juillet 2017. URL : http://conflits.revues.org/10752

Veron, Daniel (2010) « Sans-papiers : d'un quotidien tactique à l'action collective », Variations [En ligne], vol. 13/14, mis en ligne le 01 février 2012, consulté le 30 septembre 2016. URL : http:// variations.revues.org/182

Veron, Daniel (2013) « Quand les sans-papiers prennent la parole », Variations [En ligne], $\mathrm{n}^{\circ} 18$, mis en ligne le 31 mai 2013, consulté le 27 juin 2015. URL : http://variations.revues.org/641

\section{NOTES}

1. Accueil Demandeurs d'Asile (ADA) a été créée en 1986 et se consacre à l'accompagnement juridique des personnes en demande d'asile pour toutes les situations de la procédure non prises en charge par un dispositif de l'État ; sa seconde mission est la défense du droit d'asile. https:// ada-grenoble.org/

2. Voir intitulé du cahier de prison $n^{\circ} 25$ de Gramsci «Aux marges de l'histoire. Historiographie des groupes sociaux subalternes", in Antonio Gramsci, Cahiers de prison, Gallimard, Paris, 1978-1992, 5 tomes

3. Un des objectifs du groupe des Subaltern Studies, dont il est l'un des pilier, est de défier le biais élitiste de l'historiographie et de documenter l'histoire des subalternes; Elementary Aspects of Peasant Insurgency in Colonial India, Oxford University Press, Delhi, 1983, New edition: Duke Univ Press, 1999

4. L'atelier radio, co-animé avec Séréna Naudin, nourrit la méthode des espaces de parole de Modus Operandi : https://www.modop.org/espaces-de-parole/ et a donné lieu à la publication de Karine Gatelier et Séréna Naudin, «Du récit inaudible à la prise de parole : Expérience d'un atelier radio avec des personnes à la recherche d'un refuge en France ", Mémoire(s), identité(s), marginalité(s) dans le monde occidental contemporain [Online], $22 \mid 2020$, Online since 02 September 2020, connection on 07 November 2020. URL : http://journals.openedition.org/mimmoc/4043 ; DOI : https://doi.org/10.4000/mimmoc.4043

5. Les notes d'entretiens à l'OFPRA montrent qu'en moyenne les personnes disposent d'environ cinq secondes pour répondre aux questions. Notes d'entretien, Grenoble, mai 2018

6. Observation personnelle sur les expériences des personnes et les compte-rendus d'entretien de l'OFPRA. Le planning de l'OFPRA fonctionne sur la base de deux plages de rendez-vous par demijournée. Seulement dans les cas identifiés comme particulièrement complexes, l'officier peut réserver deux plages horaires, soit une demi-journée pour un entretien.

7. Légalement, les personnes doivent envoyer leur dossier de demande d'asile à l'office français de Protection des réfugiés et des Apatrides (OFPRA) 21 jours après leur passage au guichet de la préfecture. Ce passage intervient quelques jours ou quelques mois après leur arrivée en France en fonction des délais du moment, différents dans chaque région. Une fois ce dossier envoyé à l'OFPRA, les personnes attendent d'être convoquées en entretien. Et de nouveau le délai de cette convocation est variable de quelques semaines à plusieurs mois, jusqu'à une année. Les récents gouvernements cherchent à écourter ces délais pour raccourcir la procédure. On constate que plus les personnes disposent de temps avant la rédaction du récit, plus elles sont capables d'opérer une mise en récit et une réflexivité sur ce qui leur est arrivé.

8. La figure du "faux réfugié » apparaît dans les années 1980 ; elle recoupe celle du migrant économique et du fraudeur. Elle s'est traduite dans la loi, en 1993, par la notion de «demande manifestement infondée » permettant à l'OFPRA de rejeter une demande d'asile sans convoquer la personne en entretien (Grégoire, V., 2008, p.74) 
9. Extrait de la déclaration de Pascal Brice, directeur de l'OFPRA, à la journaliste du Monde, 26 mai 2015

10. Témoignage recueilli, carnet de terrain, mai 2018 , Grenoble

11. Je traduis : «Pas besoin d'entendre ta voix alors que je peux parler de toi mieux que tu ne le peux. Pas besoin d'entendre ta voix. Raconte moi seulement ta souffrance. Je veux connaître ton histoire. Et ensuite, je te la raconterai en retour d'une nouvelle façon. Je raconterai en retour d'une façon telle qu'elle sera mienne et m'appartiendra. En te ré-écrivant, je me réécris à nouveau. Je suis toujours l'auteur, l'autorité. Je suis toujours le colonisateur, le sujet parlant et tu es maintenant au centre de mon discours ".

12. Propos recueillis dans l'atelier radio, février 2018- mieux expliquer dans l'article (méthodo) ces ateliers radio

13. « Pourquoi il ne faut pas révéler la doctrine de l'OFPRA », Médiapart, 7 février 2018

14. Terme emprunté à Sophie Wahnich qui désigne ainsi les membres de la noblesse française et de la haute bourgeoisie qui, à la veille et pendant la révolution française, se soustraient à leur classe sociale d'origine et cessent d'endosser ses intérêts pour rejoindre et défendre les intérêts du peuple. Ceux-ci ont eu un rôle capital dans le déclenchement de la révolution de 1789.

\section{RÉSUMÉS}

A partir de l'expérience d'accompagnement de personnes en demande d'asile, particulièrement de la préparation à l'entretien à l'OFPRA, cet article cherche à comprendre pourquoi certains récits restent inaudibles. En plus des constats déjà établis que le récit de l'asile est contraint et empêché par de nombreux obstacles, je montre qu'un corpus de savoir est constitué en amont de la formation du récit de l'asile et parle avant et à sa place. Celui-ci représente une violence épistémique qui dépossède certaines personnes en demande d'asile de leur parole et en fait des subalternes ne pouvant être entendus. L'existence d'une violence épistémique doit questionner nos pratiques de recherche : en effet, la formation d'un savoir hégémonique met les chercheures en position de surplomb qui en plus de reproduire les rapports de domination dans les enquêtes conduites, et donc l'ordre établi, pose des biais dans la perception des réalités sociales.

\section{INDEX}

Mots-clés : demande d'asile, OFPRA, violence épistémique, récits inaudibles

\section{AUTEUR}

\section{KARINE GATELIER}

Chargée d'action-recherche au sein de Modus Operandi

Chercheure associée à l'UMR Pacte, Université de Grenoble-Alpes

karine@modop.org 